\title{
On the Geometric Blow-Up Mechanism to Scalar Conservation Laws
}

\author{
Shikuan Mao, Yongqin Liu \\ School of Mathematics and Physics, North China Electric Power University, Beijing, China \\ Email: shikuanmao@ncepu.edu.cn,yqliu2@ncepu.edu.cn
}

Received July 4, 2013; revised August 3, 2013; accepted August 13, 2013

Copyright (c) 2013 Shikuan Mao, Yongqin Liu. This is an open access article distributed under the Creative Commons Attribution License, which permits unrestricted use, distribution, and reproduction in any medium, provided the original work is properly cited.

\begin{abstract}
The focus of this article is on the geometric mechanism for the blow-up of solutions to the initial value problem for scalar conservation laws. We prove that the sufficient and necessary condition of blow-up is the formation of characteristics envelope. Whether the solution blows up or not relates to the topology structure of a set dominated by initial data. At last we take Burger's equation as an example to verify our main theorem.
\end{abstract}

Keywords: Blow-Up; Conservation Laws; Characteristics Envelope

\section{Introduction}

In this short article we consider the blow-up phenomena to scalar conservation laws. We are interested in the blow-up mechanism of the following initial value problem

$$
\left\{\begin{array}{l}
u_{t}+f(u)_{x}=0, t>0, x \in \mathbb{R}, \\
u(0, x)=u_{0}(x), \quad x \in \mathbb{R},
\end{array}\right.
$$

where $f \in C^{2}(\mathbb{R})$, without loss of generality, we can assume that $u_{0} \in H^{2}(\mathbb{R})$, i.e., we aim at the classical solution to the Equation (1.1).

By a routine proof we know that the solution to (1.1) is the limit of the corresponding viscous approximate solutions as the viscosity coefficient tends to zero, herein we omit the proof since it is not our aim. In view of the maximum principle, we know that the $L^{\infty}$-norm of the solution is controlled by the initial data, i.e., the solution itself can never be infinity at any time. On the other hand, what we concern about is the classical solutions to (1.1). So the way blow-up happens can only be when the first spatial derivative of the solution becomes infinite at some finite time.

As is well known, the solution to (1.1) blows up once the characteristics intersect (see [1-4]). However, the reverse is not true. In fact, what is needed for the blow-up to happen is the formation of the envelopes. In order to give an explicit description to the geometric blow-up mechanics for scalar conservation laws, we write this article.
In the remaining part we will study in what case the solution blows up and at the same time the characteristics intersect, and in what case the solution blows up while no characteristics intersect simultaneously. In the last section we take the Burger's equation as an example to test and verify our main theorems by constructing certain initial data.

\section{Main Theorems and Proof}

Let us first introduce the characteristics issuing from $y$

$$
\left\{\begin{array}{l}
\frac{\mathrm{d}}{\mathrm{d} t} \varphi(t, y)=f^{\prime}(u(t, \varphi(t, y))), \quad t>0, y \in \mathbb{R}, \\
\varphi(0, y)=y, \quad y \in \mathbb{R} .
\end{array}\right.
$$

From Equation (2.1) we get that

$$
u(t, \varphi(t, y))=u_{0}(y),
$$

and that

$$
\varphi_{y}(t, y)=1+t f^{\prime \prime}\left(u_{0}(y)\right) u_{0}^{\prime}(y) .
$$

From Equation (2.2) we have that

$$
u_{x}(t, \varphi(t, y)) \varphi_{y}(t, y)=u_{0}^{\prime}(y) .
$$

Denote $x=\varphi(t, y)$ and consider the family of lines (2.1) with $y$ as parameter,

$$
x=y+t f^{\prime}\left(u_{0}(y)\right), \quad y \in \mathbb{R} .
$$

In view of the knowledge of the differential geometry, 
we know that the envelope of the family of lines (2.5), i.e. characteristics envelope is the following curve with $y$ as a parameter,

$$
\left\{\begin{array}{l}
1+t f^{\prime \prime}\left(u_{0}\right) u_{0}^{\prime}(y)=0 \\
x=y+t f^{\prime}\left(u_{0}(y)\right) .
\end{array}\right.
$$

From (2.3), (2.4) and (2.6), we have the following results.

Theorem 2.1. The solution $u$ to (1.1) blows up at time $t$ if and only if there exists some $y \in \mathbb{R}$ such that $\varphi_{y}(t, y)=0$, or equivalently the characteristics envelope forms at time $t$.

From the previous analysis, the proof of Theorem 2.1 is obvious. Moreover, under some stronger assumptions, we have more detailed results than Theorem 2.1, i.e. the following Theorem 2.2.

If $\min _{y \in \mathbb{R}}\left[f^{\prime \prime}\left(u_{0}(y)\right) u_{0}^{\prime}(y)\right]$ exists, then the concrete relation between blow-up and characteristics is determined by the topology structure of the following set

$$
\begin{aligned}
A:= & \left\{x \in \mathbb{R} f^{\prime \prime}\left(u_{0}(x)\right) u_{0}^{\prime}(x)\right. \\
& \left.=\min _{y \in \mathbb{R}}\left[f^{\prime \prime}\left(u_{0}(y)\right) u_{0}^{\prime}(y)\right]<0\right\}
\end{aligned}
$$

We denote $A^{0}$ by the set of all the inner points of $A$. Then we have the following theorem

Theorem 2.2. Assume that $\min _{y \in \mathbb{R}}\left[f^{\prime \prime}\left(u_{0}(y)\right) u_{0}^{\prime}(y)\right]$ exists and $A$ is defined as above. There exist the following three cases:

$A=\varnothing$, then the solution $u$ to Equation (1.1) exists globally.

$A \neq \varnothing, A^{0}=\varnothing$, then the solution $u$ to (1.1) blows up, while no characteristics intersect when blow-up happens.

$A^{0} \neq \varnothing$, then the solution $u$ to (1.1) blows up and at the same time the characteristics intersect.

Proof. 1) Since $A=\varnothing$, and $u_{0} \in H^{2}(\mathbb{R})$, we have $f^{\prime \prime}\left(u_{0}(x)\right) u_{0}^{\prime}(x) \geq 0, \forall x \in \mathbb{R}$.

It yields that $\varphi_{y}(t, y) \geq 1$, so $\left|u_{x}(t, \varphi(t, y))\right| \leq\left|u_{0}^{\prime}(y)\right|$, i.e. the solution to Equation (1.1) exists globally.

2) Since $A \neq \varnothing, A^{0}=\varnothing$, there exists $y_{0} \in A$. Let $t_{0}=-\left(f^{\prime \prime}\left(u_{0}\left(y_{0}\right)\right) u_{0}^{\prime}\left(y_{0}\right)\right)^{-1}$, then $\varphi_{y}\left(t_{0}, y_{0}\right)=0$. From (2.4) and Theorem 2.1, $u_{x}\left(t_{0}, \varphi\left(t_{0}, y_{0}\right)\right)=\infty$, so the solution $u$ blows up and $t_{0}$ is the maximal existence time of the smooth solution.

Now we prove that no characteristics intersect at time $t_{0}$ by contradiction. Suppose that there are two characteristics issuing from $y_{1}, y_{2}$, where $y_{1}<y_{2}$, such that $\varphi\left(t_{0}, y_{1}\right)=\varphi\left(t_{0}, y_{2}\right)$. Since $t_{0}=-\left[f^{\prime \prime}\left(u_{0}\left(y_{0}\right)\right) u_{0}^{\prime}\left(y_{0}\right)\right]^{-1}=-\left(\min _{y \in \mathbb{R}}\left[f^{\prime \prime}\left(u_{0}(y)\right) u_{0}^{\prime}(y)\right]\right)^{-1}$,

we have that $\varphi_{y}\left(t_{0}, y\right) \geq 0$, and this results in $\varphi\left(t_{0}, y\right)=\varphi\left(t_{0}, y_{1}\right), \forall y \in\left[y_{1}, y_{2}\right]$. Then we have that $\varphi_{y}\left(t_{0}, y\right)=0, \forall y \in\left(y_{1}, y_{2}\right)$, i.e.

$$
f^{\prime \prime}\left(u_{0}(y)\right) u_{0}^{\prime}(y)=-\frac{1}{t_{0}}=f^{\prime \prime}\left(u_{0}\left(y_{0}\right)\right) u_{0}^{\prime}\left(y_{0}\right), \forall y \in\left(y_{1}, y_{2}\right) \text {. }
$$

It yields that $y_{0} \in A^{0}$. This contradicts with the assumption that $A^{0}=\varnothing$. So the characteristics do not intersect when the blow-up happens.

3) Since $A^{0} \neq \varnothing$, choose $y_{0} \in A^{0}$. Let

$t_{0}=-\left(f^{\prime \prime}\left(u_{0}\left(y_{0}\right)\right) u_{0}^{\prime}\left(y_{0}\right)\right)^{-1}$, then $\varphi_{y}\left(t_{0}, y_{0}\right)=0$. From (2.4) and Theorem 2.1, $u_{x}\left(t_{0}, \varphi\left(t_{0}, y_{0}\right)\right)=\infty$, so the solution $u$ blows up and $t_{0}$ is the maximal existence time of the smooth solution.

Now we prove that the characteristics intersect at time $t_{0}$. Since $y_{0} \in A^{0}$, then there exist $y_{1}, y_{2} \in \mathbb{R}$ such that $\left[y_{1}, y_{2}\right] \subset A^{0}$, i.e.

$$
f^{\prime \prime}\left(u_{0}(y)\right) u_{0}^{\prime}(y)=f^{\prime \prime}\left(u_{0}\left(y_{0}\right)\right) u_{0}^{\prime}\left(y_{0}\right)<0, \forall y \in\left[y_{1}, y_{2}\right] \text {. }
$$

It yields that $f^{\prime}\left(u_{0}(y)\right)$ is a line segment on $\left[y_{1}, y_{2}\right]$ and

$$
\varphi_{y}\left(t_{0}, y\right)=\varphi_{y}\left(t_{0}, y_{0}\right)=0, \forall y \in\left[y_{1}, y_{2}\right] . .
$$

So, $\varphi\left(t_{0}, y\right)=\varphi\left(t_{0}, y_{0}\right), \forall y \in\left[y_{1}, y_{2}\right]$, i.e. the characteristics intersect.

Remark 1). The solution to Equation (1.1) exists globally if $f^{\prime}\left(u_{0}(y)\right)$ is a nondecreasing function of $y \in \mathbb{R}$.

2) The solution $u$ blows up first along the characteristics which issue from the points where $f^{\prime}\left(u_{0}(y)\right)$ decreases fastest.

3) If the set of the points where $f^{\prime}\left(u_{0}(y)\right)$ decreases fastest contain an inner point, some characteristics intersect and the solution blows up. Otherwise, the characteristics do not intersect while the solution still blows up by reason of the formation of the envelope so long as $f^{\prime}\left(u_{0}(y)\right)$ decrease in a neighborhood of some point.

\section{Burger's Equation}

In this part, we take Burger's equation as an example to testify Theorem 2.2. The initial value problem for Burger's equation is the following

$$
\left\{\begin{array}{l}
u_{t}+u u_{x}=0, \quad t>0, x \in \mathbb{R}, \\
u(0, x)=u_{0}(x), \quad x \in \mathbb{R},
\end{array}\right.
$$

which is equivalent to (1.1) with $f(u)=u^{2}$. The corresponding set $A$ is as follows with the assumption that $\min _{y \in \mathbb{R}} u_{0}^{\prime}(y)$ exists, 


$$
A=\left\{x \in \mathbb{R} ; u_{0}^{\prime}(x)=\min _{y \in \mathbb{R}} u_{0}^{\prime}(y)<0\right\} .
$$

From Theorem 2.2, we know that if there is some point on which $u_{0}^{\prime}$ is negative, then the solution to equation (3.1) blows up along the characteristics which issues from the point. And if the picture of $u_{0}(y), y \in \mathbb{R}$ restricted on the set of the points on which $u_{0}^{\prime}$ is negative does not contain any line segments, then no characteristics intersect, but the solution to Equation (3.1) still blows up along these characteristics issuing from those points on which $u_{0}^{\prime}$ is negative.

Now we give certain initial data for Burger's equation corresponding to the case 2) in Theorem 2.2. We take $u_{0}$ as follows

$$
u_{0}(x)=\left\{\begin{array}{lr}
0, & |x|>2, \\
(x+2)^{2}, & -2 \leq x \leq-1, \\
2-x^{2}, & -1<x<1 \\
(x-2)^{2}, & 1 \leq x \leq 2 .
\end{array}\right.
$$

Then we have that

$$
u_{0}^{\prime}(x)=\left\{\begin{array}{lr}
0, & |x|>2, \\
2(x+2), & -2 \leq x \leq-1, \\
-2 x, & -1<x<1, \\
2(x-2), & 1 \leq x \leq 2 .
\end{array}\right.
$$

It is easy to see that $u_{0} \in H^{2}(\mathbb{R})$, and $\min _{x \in \mathbb{R}} u_{0}^{\prime}(x)=u_{0}^{\prime}(1)=-2$. The characteristics issuing from $x_{0}$ can be described as $x=x_{0}+t u_{0}\left(x_{0}\right)$, taking $x_{0}=1$, we get that $x=1+t$. By the computation similar to the proof of Theorem 2.2, we know that the maximal existence time of the solution is

$t_{0}=-\left(\min _{x \in \mathbb{R}} u_{0}^{\prime}(x)\right)^{-1}=\frac{1}{2}, \quad$ and $\quad u_{x}\left(\frac{1}{2}, \frac{3}{2}\right)=-\infty$. How ever, there is no characteristics intersecting at time $t=\frac{1}{2}$ when blow-up happens.

Next we give certain initial data for Burger's equation corresponding to the case 3) in Theorem 2.2. Assume that $u_{0}$ is as follows,

$$
u_{0}(x)=\left\{\begin{array}{lr}
0, & x<-2, \\
(x+2)^{2}, & -2 \leq x \leq-1 \\
2-x^{2}, & -1<x<1 \\
3-2 x, & 1 \leq x \leq 2 \\
(x-3)^{2}-2, & 2<x<4 \\
-(x-5)^{2}, & 4 \leq x \leq 5 \\
0, & x>5
\end{array}\right.
$$

Then we have that

$$
u_{0}^{\prime}(x)=\left\{\begin{array}{lr}
0, & x<-2, \\
2(x+2), & -2 \leq x \leq-1, \\
-2 x, & -1<x<1, \\
-2, & 1 \leq x \leq 2, \\
2(x-3), & 2<x<4, \\
-2(x-5), & 4 \leq x \leq 5, \\
0, & x>5 .
\end{array}\right.
$$

It is easy to see that $u_{0} \in H^{2}(\mathbb{R})$, and $\min _{x \in \mathbb{R}} u_{0}^{\prime}(x)=u_{0}^{\prime}\left(x_{1}\right)=-2$, for any $x_{1} \in[1,2]$. The equation for the characteristics issuing from $x_{0}$ is $x=x_{0}+t u_{0}\left(x_{0}\right)$, taking $x_{0}=x_{1} \in[1,2]$, we get that $x=x_{1}+t\left(3-2 x_{1}\right)$. By the computation similar to the proof of Theorem 2.2, we know that the maximal existence time of the solution is $t_{0}=-\left(\min _{x \in \mathbb{R}} u_{0}^{\prime}(x)\right)^{-1}=\frac{1}{2}$, and $u_{x}\left(\frac{1}{2}, \frac{3}{2}\right)=-\infty$. Moreover at time $t=\frac{1}{2}$, we have that $x=x_{1}+\frac{1}{2}\left(3-2 x_{1}\right)=\frac{3}{2}, \forall x_{1} \in[1,2]$, i.e., the characteristics issuing from the points in [1,2] intersect at the point $\left(\frac{1}{2}, \frac{3}{2}\right)$ in the $(t, x)$-plane as blow-up happens.

\section{Acknowledgements}

The authors would give thanks to Professor Weike Wang for the helpful discussion. The first author is partially supported by the National Natural Science Foundation of China (Grant No. 11201142) and by the Fundamental Research Funds for the Central Universities (Grant no. 11QL40). The second author is partially supported by the National Natural Science Foundation of China (Grant No. 11201144).

\section{REFERENCES}

[1] S. Alinhac, "Blowup for Nonlinear Hyperbolic Equations,” Birkhäusera, Boston, 1995. doi:10.1007/978-1-4612-2578-2

[2] F. John, "Nonlinear Wave Equations, Formation of Singularities," American Mathematical Society, Providence, 1990.

[3] H. A. Levine and M. H. Protter, "The Breakdown of Solutions of Quasilinear First Order Systems of Partial Differential Equations," Archive for Rational Mechanics and Analysis, Vol. 95, No. 3, 1986, pp. 253-267. doi:10.1007/BF00251361

[4] J. Smoller, "Shock Waves and Reaction-Diffusion Equations,” Springer Verlag, New York, Heidelberg, Berlin, 1983. doi:10.1007/978-1-4684-0152-3 